\title{
Failure of familiar size to determine a metric for visually perceived distance
}

\author{
DONALD H. MERSHON \\ North Carolina State University, Raleigh, North Carolina 27607 \\ and \\ WALTER C. GOGEL \\ University of California, Santa Barbara, California 93106
}

\begin{abstract}
The use of knowledge of the familiar sizes of objects in determining the apparent distances of those objects is known as the familiar size cue to distance. If effective, this cue might be one of the factors responsible for supplying the metric (scalar) characteristics of perceptions of spatial extent within a visual display in which other information concerning scalar extents has been reduced to a minimum. Two groups of observers were presented with realistic objects of the same angular, but different assumed, sizes presented in such a cue-restricted display. Perceptions of size and distance within the display did not differ significantly as a function of the type of object initially presented. This result was consistent with the notion that scalar perceptions under these conditions probably are determined by a factor known as the specific distance tendency, rather than by the experiential factor of familiar size.
\end{abstract}

The use of the familiar or assumed size of an object to determine the apparent distance of that object from the observer is the familiar size cue to egocentric distance. Familiar size represents the clearest situation in which experiential factors may be involved in space perception and has generated much experimental interst (cf. Schiffman, 1967; Slack, 1956). In general, it has been concluded that familiar size may be an independent variable in the perception of relative (exocentric) distances (Gogel \& Mertens, 1968; Newnan. 1972; Ono, 1969), but is probably not an important factor in the perception of (as contrasted with the cognitive response to) egocentric distances (Gogel \& Newton, 1969). In addition, it has been asserted that the form of the instructions used, e.g., an emphasis upon estimations of "real" sizes and distances, may lead to reports which, although they reflect an effect of familiar size, do so by producing cognitive judgments without influencing directly the perception of extents (Gogel, 1969a).

There is some difficulty in obtaining estimates of the apparent sizes and distances of familiar objects without the intrusion of cognitive biases. If one merely asks how large a familiar object is, the observer may report either its apparent size or its assumed (cognitive) size, if these differ, or some combination of born. Similarly, either or both apparent and cognitive effects can be involved in reports of distance. If one asks merely how far away the object is, the response

This study was conducted while the first author was a graduate student at the University of California, Santa Barbara, and was supported by PHS Research Grant No. 15651 from the National Institute of Mental Health, awarded to the second author. may be determined by some combination of the genuine apparent egocentric distance to the object (which may make the object appear to be different in size than its expected familiar size) and the observer's cognitive estimate of the object's distance. If the object appears "too small," for example, the observer may report it as being far away due to a bias that "small" and "far away" are synonymous (Carlson, 1960). Obviously, it is important in any study in which apparent extents are to be measured to avoid as much as possible the intrusion of cognitive factors. For this reason, in the present study, direct scalar reports about the perceived sizes and perceived egocentric distances of the familiar objects were avoided, and instead these perceptions were measured in a somewhat less direct manner.

The study of egocentric distance perception is closely tied to the question of how visual perceptions of space become endowed with metric (scalar) characteristics (Gogel, 1968). The fact that a visual display may be perceptually specific with regard to the relative positions and sizes of objects indicates nothing concerning the metric magnitudes of the perceived sizes and distances. For example, if all extents in the display are seen to be twice as large, or 20 times as large, or half as large in one case compared to another, the relative, although not the metric, perceived extents would remain unchanged. The factors determining scalar perceptions of extent are not identical to those involved in the perception of relative extents (Gogel, 1972).

Consider, for example, a display consisting of a checkerboard pattern of alternating black-and-white 
squares receding in depth from the observer, with the nearest edge of the display at some distance in front of the observer. Within the display are numerous cues of exocentric distance, such as perspective, to maintain the perception of a surface extended in depth. But, under the proper viewing conditions (monocular observation through a restrictive aperture), the available information concerning the egocentric distances of the parts of the display is severely reduced if not eliminated. Nevertheless, even under such impoverished conditions, the observers are capable of responding with scalar judgments of both egocentric distance and of extents within the display, although neither kind of perception is necessarily veridical.

The ability of the observer to produce scalar responses under reduced conditions of observation indicates that familiar sizes are not necessary in order for scalar perceptions to occur. It does not indicate, however, that familiar size is unable to provide a metric for calibrating a visual scene. The purpose of the present study was to examine whether the introduction of an object having a familiar size would calibrate egocentric distance within a visual field in which conventional egocentric cues to distance were absent. One method of examining the possible role of familiarity in this process is to provide, on different occasions, familiar objects of the same shape and retinal size but with different familiar sizes. If familiarity is effective in calibrating the perception of egocentric extents within the display, it should be found that the perceived egocentric distances will be directly related to the assumed size of the familiar object, with retinal size of the familiar object constant. To provide the condition of a constant retinal size but different assumed sizes, physically off-sized presentations of familiar objects are necessary. For this purpose, back-illuminated transparencies of a stamp or a university catalog were used in the present study.

If the familiar size of an object is unable to influence the perceptual scale, then the scale of the display will be determined only by whatever other sources of scalar information may be present in the situation. One such factor which can influence scalar perceptions of extent is the specific distance tendency (SDT). This is the tendency for an object to appear about $2 \mathrm{~m}$ from the observer in the absence of effective egocentric cues to distance (Gogel, 1969b, 1972). In a situation such as the present one, this tendency can act as a reference distance to part of the display and can thereby provide a ruler for metric perceptions throughout the display. The scalar values produced by this factor, since they are not determined by external stimulation are not expected to differ as a function of the identity of the familiar object presented in the display.

\section{METHOD}

\section{Apparatus}

The apparatus consisted of a small rectangular room, $34.3 \mathrm{~cm}$ high, $55.9 \mathrm{~cm}$ wide, and $62.2 \mathrm{~cm}$ deep, which was viewed monocularly by the observer (with his right eye) through a $1-\mathrm{mm}$ restrictive aperture at an observation position located $20.8 \mathrm{~cm}$ in front of the near wall of the room and centered with respect to that wall. The far wall of the room, made of translucent material, was transilluminated by fluorescent light to a luminance of $64.6 \mathrm{~mL}$. The far wall contained two windows $(14 \mathrm{~cm}$ square $)$ separated laterally by $7.3 \mathrm{~cm}$. Behind each window was a hollow enclosure extending approximately $45.7 \mathrm{~cm}$ in depth along lines of sight from the observer. These enclosures permitted stimuli to be presented either immediately behind the windows $(85.7 \mathrm{~cm}$ from the observer's eye) or $42.5 \mathrm{~cm}$ behind the windows, while remaining directionally centered in the windows. The stimulus objects were high-quality color transparencies of a current university catalog or a current U.S. commemorative postage stamp mounted on cardboard carricrs. The physical (familiar) horizontal extents of the objects photographed were $2.5 \mathrm{~cm}$ for the stamp and $14.9 \mathrm{~cm}$ for the callalog. The transparencies of the objects were photographically enlarged (stamps) or reduced (catalogs) so that the horizontal dimension of each was $5.0 \mathrm{~cm}$. The vertical dimensions of the transparencies were also approximately equal $(8.0$ and $7.6 \mathrm{~cm}$ for the stamp and catalog, respectively). The transparencies were transilluminated by another diffuse, homogeneously illuminated surface to a luminance of approximately $40 \mathrm{~mL}$. The background on which the transparencies were mounted was opaque, so that each familiar object appeared to be suspended in the middle of a square black window (see Figure 1). The floor of the room was composed of a checkerboard pattern of black and white squares $(5.1 \mathrm{~cm}$ on a side), with the walls and ceiling light gray.

When the observer looked into the room, he perceived a rectangular room with a checkerboard floor and a white far wall on which were located two black square windows, with each containing a familiar object (transparency). The familiar objects, one in each window, were both of the same kind, i.e., no observer was ever simultaneously presented with both a stamp and a catalog. Two vertical markers beneath the windows at the base of the far wall were adjustable by the observer in lateral separation by turning a handle located at the observation position. The adjusted separation of the markers in centimeters was read by the experinenter from a scale located outside the room and invisible to the observer.

The observation position contained an adjustable chinrest, the restrictive aperture, the handle for moving the markers, and a communication system, and was located in a booth which could be made totally dark. A shutter occluded the observer's view of the display between presentations.

\section{Subjects}

Forty students from a course in introductory psychology served as observers. They were between the ages of 17 and 24 years (median age 18) and had a visual acuity of at least $20 / 20$ in the right eye (corrected with contact lenses, if necessary) as measured by a Keystone orthoscope.

\section{Procedure}

On the first trial, half of the observers were presented with two stamps at the same distance from the observer, with one stamp positioned immediately behind each of the windows. The remaining observers were presented with two catalogs at the same distance from the observer, with one catalog positioned immediately behind each of the windows. Each observer indicated the perceived width (left to right extent) of one of the familiar objects by adjusting the lateral separation between the black markers at the base of the far wall (see Figure 1) until this separation appeared to be the same as the width of the transparency. For half the observers, the starting 


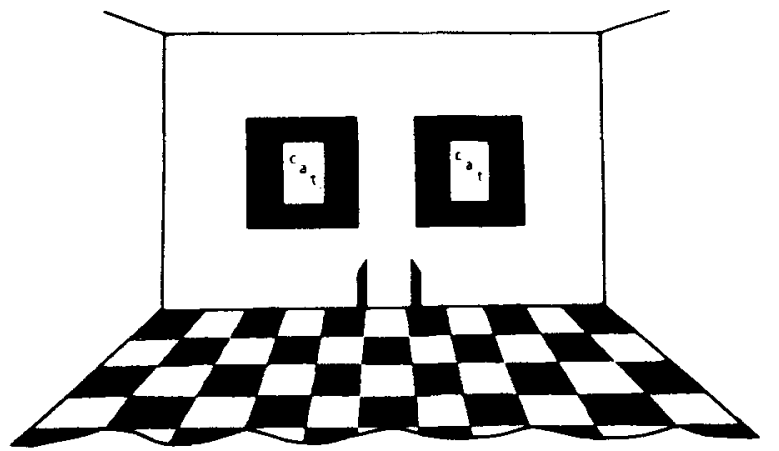

Figure 1. Observer's view of the display, as presented with two current university catalogs (transparencies) of the same size in the two windows. In the experiment itself, the transparencies (either two stamps or two catalogs) were observed in full color reproduction. The vertical markers used to measure the perceived sizes of the stamps or catalogs were located below the windows as shown. See text for other details of construction and procedure.

position of the markers was always together; for half, they were always separated. Following this, the observer indicated verbally in feet or inches or some combination of feet and inches the apparent depth between the two familiar objects and between each familiat object and its surrounding window. Using the black markers, the observer then indicated the apparent width of the other familiar object.

Since the first judgments of size involved a perception of relative size, i.e., the perceived size of the familiar object relative to the perceived separation of the black markers, additional information was required to convert these relative perceptions of size to scalar values. This was accomplished by having the observer adjust the black markers until they appeared to be separated by $3 / 4$ or $1 \frac{1}{2}$ in. (on alternate trials). Finally, the observer reported verbally in feet and inches the apparent distance between himself and the base of the far wall located between the markers. This last judgment completed the first trial.

Following the first trial, the observer was presented with two trials in which the same transparency (the stamp or catalog) again was in both windows. In these trials, however, one transparency was located immediately behind its window $(85.7 \mathrm{~cm}$ from the observer's eye), whereas the other was located $42.5 \mathrm{~cm}$ behind the window (128.2 cm from the observer's eye). Thus, in the second and third trial, as in the first trial, familiar objects of the same type and physical size were presented, one in each window. But, unlike the first trial, the retinal sizes of the two familiar objects were different with the near object having a larger retinal size than the far object. The same kind of judgments of size and distance were made as in the first trial. The second and third trial differed only in whether the near or far transparency was in the left or right window.

Following the third trial, each observer was presented with three additional trials, which were identical to the three trials described above, except that the familiar objects (stamps or catalogs) not previously used for that observer were presented, one in each window. As before, the condition in which the two identical transparencies were at the same distance from the observer was presented before presenting the condition in which they were at different distances.

After completion of all six trials, the shutter was again closed and the observer was asked to report verbally the remembered width, in inches, of an actual university catalog and an actual U.S. postage stamp (of the types used in the experiment).

\section{RESULTS}

\section{Data from First Trials}

For the purpose of the present study, the most
Table 1

Data from First Trial: Perceived Distance $\left(D^{\prime}\right)$ to Far Wall, Perceived Depth (d $\left.d^{\prime}\right)$ Between Familiar Objects and Between Objects and Surrounding Windows, Perceived Relative Size Matches of Markers to the Familiar Objects, Perceived Scalar Size $\left(S^{\prime}\right)$ of Familiar Objects, and Judgments from Memory (Assumed Size) of Familiar Objects, With All Values Converted to Centimeters

\begin{tabular}{llrrr} 
& & Stamps & Catalogs & \\
\hline & Mean & 238.2 & 226.8 & \\
$\mathrm{D}^{\prime}$ & SD & 166.6 & 134.6 & \\
& Median & 274.3 & 222.2 & n.s.* $^{*}$ \\
d' $^{\prime}$ & Mean & -1.6 & .8 & \\
(Between & SD & 9.2 & 12.3 & \\
familiar & Median & .0 & .0 & \\
objects) & InterQ. Range & 1.2 & .0 & \\
d' (Between & Mean & -.4 & 4.2 & \\
familiar & SD & 7.2 & 11.6 & \\
object and & Median & .0 & .0 & \\
window) & InterQ. Range & .0 & 6.0 & \\
Relative Size & Mean & 5.0 & 5.7 & \\
(Uncali- & SD & .7 & 1.0 & \\
brated) & Median & 4.8 & 5.5 & p $<.02^{*}$ \\
& Mean & 14.6 & 13.6 & \\
S' & SD & 18.5 & 10.4 & \\
& Median & 3.1 & 12.7 & n.s.* \\
& Mean & 2.0 & 15.6 & \\
Assumed & SD & .7 & 3.2 & \\
Size & Median & 1.9 & 15.2 & p $<.001^{*}$ \\
\hline
\end{tabular}

${ }^{*}$ Differences in $D^{\prime}$, relative size, $S^{\prime}$, and assumed size tested by Mann-Whitney $U$ test.

relevant data are the results from the first trials for each of the two groups of observers. These are shown in Table 1, along with the judgments of the remembered sizes of the real familiar objects. Since the results from judgments of size and distance often tend to be skewed, both mean and median values are given and all statistical tests are nonparametric.

In the upper half of Table $1 \mathrm{D}^{\prime}$ is the average verbal report of the perceived distance (converted to centimeters) of the far wall from the observer, and the results labeled $d^{\prime}$ are the verbal reports of the perceived depth (also converted to centimeters) between the familiar objects or between these objects and their surrounding windows. In the lower half of Table 1, three types of size report are shown. The results labeled "Relative Size" represent the actual lateral separation of the black markers when the observers adjusted them to match the apparent width of each of the objects (transparencies). The data labeled $S^{\prime}$ are the perceived scalar widths of these same objects. The $S^{\prime}$ values were obtained by applying the lateral separation of the black markers adjusted by the observer to the remembered widths of $3 / 4$ or $1 \frac{1 / 2}{}$ in. as a calibration for the above measures of relative matched size produced by the same observer. For example, if a particular observer had produced a physical separation of the black markers of $1 / 2$ in., when instructed to duplicate $1 \frac{1}{2} \mathrm{in}$., the adjusted 
relative size width for the familiar object for that observer would have been multiplied by 3 , i.e., by $1.5 / 0.5$, to obtain $S^{\prime}$. The resulting values of $S^{\prime}$ obtained from the two same familiar objects on the first trial are combined in Table 1. since no consistent difference was obtained between left and right positions. The results labeled "Assumed Size" are the verbal reports of the remembered sizes of actual (normally encountered) stamps and catalogs (converted to centimeters) obtained at the end of the trials.

If the familiar sizes of the transparencies had determined the metric perceptions of width, $S^{\prime}$ in Table 1 would have been $2.5 \mathrm{~cm}$ for the stamps and $14.9 \mathrm{~cm}$ for the catalogs. If the familiar sizes of the transparencies had determined the metric perceptions of the distance of the back wall, $\mathrm{D}^{\prime}$ in Table 1 (as calculated from the familiar size, and the physical size and distance of the transparencies) would have been $43 \mathrm{~cm}$ when using the stamps and $256 \mathrm{~cm}$ when using the catalogs. It will be noted in Table 1 that neither $S^{\prime}$ nor $D^{\prime}$ differed significantly for the stamps as compared with the catalogs, although there was some tendency for the median $S^{\prime}$ values (as well as the uncalibrated size measures) to be larger for the catalogs than for the stamps. Since this latter result was not reflected in a difference in $\mathrm{D}^{\prime}$, as should have occurred according to the size-distance invariance hypothesis, it probably represents a cognitive rather than a perceptual difference. Also, it will be noted from the d' data of Table 1 that each familiar object appeared to be located at the distance of its respective window. This tendency to perceive the familiar object at the distance of the window in which it appears is consistent with the equidistance tendency (EDT) which states that under reduced cues to depth there is a tendency for objects to appear at the same distance from the observer (Gogel, 1965). Generalizing the D' and, to a lesser extent, the $S^{\prime}$ results to other portions of the room, it can be concluded that the perceived metric sizes and distances of the room were approximately the same regardless of the familiar size of the transparencies presented in the windows.

\section{Data from Later Trials}

When presented with the same type of familiar object as on the first trial, but with the two familiar objects at different distances behind the windows, again each transparency usually appeared to be at or very near the distance of its enclosing window. Median d'values were 0.0 and $5.2 \mathrm{~cm}$ for the near and far catalog and 0.0 and $0.0 \mathrm{~cm}$ for the near and far stamp, respectively. Although there were some differences in $\mathrm{D}^{\prime}$ among the trials for which the familiar objects were at different physical distances, the interpretation of these differences is complex.

The median $S^{\prime}$ values were 12.0 and $7.9 \mathrm{~cm}$ for the near and far catalog and 3.5 and $2.3 \mathrm{~cm}$ for the near and far stamp respectively. These differences in perceived size, due to the EDT making the familiar objects appear at the same distance, despite their difference in physical distance, are consistent with the results obtained from geometrical objects in the study of Gogel and Mershon (1968).

Half of the observers first observed three presentations of the stamps, and the other half first observed three presentations of the catalogs. It is of some interest to determine whether the change from one familiar object to another for the same observers affected the perceived metric characteristics of the room. For observers seeing first stamps then catalogs, there was no change in median $\mathrm{D}^{\prime}$ between the two conditions with two equal retinal sizes of these familiar objects (Trial 1 vs. Trial 4). For observers seeing first catalogs then stamps, there was some tendency to see the catalogs as being in a slightly larger room than the stamps (median $D^{\prime}$ values of 222.2 and $191.7 \mathrm{~cm}$, respectively, $p<.01$ by sign test). If it is assumed that the response to the distance of the far wall in the case of these successive presentations is indeed perceptual (not cognitive), these results constitute weak evidence that some effect upon the perceived metric characteristics of the room can occur as a function of a change in the metric (the assumed size per unit of retinal size) of the familiar object between successive presentations. Only the initial presentations, of course, speak to the question of whether familiar size as a possible cue to egocentric distance can serve to establish a metric for perceived extents within a visual scene (cf. Gogel, 1968).

\section{DISCUSSION}

If the familiar size of the transparency had determined the perceived metric of the room, the room should have been perceived to be about 6 times as large when the catalogs, rather than the stamps, were presented in the windows. Clearly, rather than a change of this magnitude, little, if any, scalar change occurred when the different familiar objects were used. Two related questions seem appropriate to ask of the study: (1) What were the factors which determined the perceived metric or scale of the room, and (2) why did a change in familiarity not result in a change in the scale?

It was noted that if the scale of the room had been consistent with the familiar sizes, $S^{\prime}$ and $D^{\prime}$ in Table 1 should have been 2.5 and $43 \mathrm{~cm}$ with the stamps and 14.9 and $256 \mathrm{~cm}$ with the catalogs, respectively. The $\mathrm{D}^{\prime}$ results in Table 1 are clearly more consistent with those expected from the catalogs than from the stamps. But these results are also consistent with those expected from the specific distance tendency (SDT). It has been found, with a display consisting of 
two points of light separated in apparent depth, that the more distant portion of the display tends to be perceived near, but somewhat beyond, the distance defined by the specific distance tendency (Gogel, 1972). Since the far wall is perceptually the most distant portion of the room, it is likely from the SDT that the far wall will appear to be at least $2 \mathrm{~m}$ from the observer. Another factor that could have contributed to the scale of perceived extent within the room is the range of perceived sizes of rooms usually encountered by the observer. The median values of $D^{\prime}$ in Table 1 would indicate a rather small room (about $9 \mathrm{ft}$ with the stamps and $7 \mathrm{ft}$ with the catalogs). But there is evidence that verbal reports of egocentric distances tend to be underestimations of the distances actually perceived (Gogel, 1968, 1969a), so that the perceived depth of the room is likely to be larger than that reported, and more similar to the dimensions of normally encountered rooms.

Of the three possible factors, the familiar sizes of the stamp and catalog transparencies, the familiar size of a room, and the SDT, the last is the most likely to have determined the perceived scale of the room. The reasons for this conclusion are as follows. It seems reasonable to expect that the more unique the size of a familiar object, the more that familiar size would be expected to contribute to the perception. Unlike either the postage stamps or catalogs used in the study, rooms exist in a wide variety of sizes. Past experience as to the size of a room would be much more variable and, hence, much less useful in specifying a scale, than past experience with respect to the stamps or catalogs. Thus the familiar size of stamps or catalogs would be expected to be more effective than the familiar size of a room in determining perceived scale in this study. But, since the present results provide little or no support for a change in the perceived scale with a sixfold change in the familiar size of the transparencies, it seems unlikely that this scale can be attributed to the familiar size of either transparency (and even more unlikely that it can be attributed to the more variable size of rooms in general). It follows that the most likely explanation of the responses on the first trial is that the transparencies appeared at the distance of the windows on the back wall as a result of the EDT, the perceived location of the back wall (and the transparencies) was determined by the SDT, and the perceived sizes of the transparencies were determined by their retinal sizes and perceived distances via the size-distance invariance hypothesis (SDIH).

If the above interpretation of the results is valid, the question occurs as to why the familiar sizes of the transparencies failed to provide a scale for perceived extent within the room. There are two possible answers to this question. One answer, favored by the authors, is that familiar size can determine a cognitive but not a perceived size. This point of view would assert that familiar size does not determine either perceived size or perceived distance, with the result that in the present study these characteristics are determined by other factors such as the EDT, SDT, and SDIH.

A second, different, answer would be that the study failed to provide a fair test of the role of familiar size, since (as indicated in Table 1) the familiar objects often were not perceived to be normal in size. This point of view would assert that a familiar object must appear normal in size if its familiarity is to be effective in determining a perceptual scale. The present lack of perceived normality of the familiar objects might be due either to the objects not being familiar to the observer or to the presence of "give away" cues in the room to indicate to the observer that the objects were only transparencies.

The failure of familiar (assumed) size to determine $S^{\prime}$ or to provide a metric for the calibration of the perceived distance to the far wall cannot be attributed to any lack of information regarding the normal sizes of the familiar objects, since no overlap was present in the distributions of the remembered sizes of the stamps and catalogs. The median values of the postexperimental judgments of the width of a real stamp and catalog (see Table 1) are similar to the naturally occurring physical widths of the real stamp and catalog $(2.5$ and $14.9 \mathrm{~cm})$ and differed significantly at the .001 level. Although there clearly was a fairly accurate memory of the familiar sizes of the stamps and catalogs, this information on the first trials did not determine perceived distance and probably did not determine perceived size.

Nor can the failure of familiar size to determine $S^{\prime}$ or $D^{\prime}$ be attributed to cues indicating that transparencies rather than real objects were being used. If such "give away" cues had been present, the $S^{\prime}$ values should have corresponded to the $5-\mathrm{cm}$ physical width of the transparencies. Clearly, this was not the case. $^{b}$ It is correct, "of course, that the objects (particularly the stamps) often were not seen as normal in size. This frequent failure of familiar size to determine perceived size must be attributed to the failure of familiar size to determine the perceived scale of the room. Because the perceived scale of the room (as determined by the SDT and SDIH) was considerably larger than the scale consistent with the familiar size of the stamps, the stamps appeared much larger than their normal size. Thus, the argument that an adequate test of familiarity as a scaling factor requires that the familiar objects appear normal in size is obviously circular. Any failure of familiar size to determine a scale consistent with that familiar size must lead to distortions in the perceived size of the familiar object. If such a circular criterion were applied, only positive results would be accepted 
as an adequate test of familiarity as a scaling factor, in which case there would be no reason to do the research, since it would have no possibility of failure.

As an additional alternative explanation, it might be argued that if the SDT had not been present, the change in familiar size would have resulted in a change in the perceived scale of the room. But, since the SDT is always potentially present, any determiner of perceived scale must be able to modify the scale effect of the SDT.

In general, the results from the study do not offer much comfort for the notion that familiarity provides a scale for visual fields. The question of how a scale is introduced into a visual field, if not by familiarity, is important. Although an answer in terms of the SDT, EDT, and SDIH is at present speculative, it seems to the authors to be an approach consistent with the present data.

\section{REFERENCES}

Carlson, V. R. Overestimation in size-constancy judgments. American Journal of Psychology, 1960, 73, 199-213.

Carlson, V. R., \& Tassone, E. P. Familiar versus unfamiliar size: A theoretical derivation and test. Journal of Experimental Psychology, 1971, 87, 109-115.

GoGEL, W. C. Equidistance tendency and its consequences. Psychological Bulletin, 1965, 64, 153-163.

GogEL, W. C. The measurement of perceived size and distance. In W. D. Neff (Ed.), Contributions to sensory physiology (Vol. 3). New York: Academic Press, 1968. Pp: 125-148.

GoGel, W. C. The effect of object familiarity on the perception of size and distance. Quarterly Journal of Experimental Psychology, 1969, 21, 239-247. (a)

Gogel, W. C. The sensing of retinal size. Vision Research, 1969, 9, 3-24. (b)
Gogel, W. C. Scalar perceptions with binocular cues of distance. American Journal of Psychology, 1972, 85, 477-497.

GOGEL, W. C., \& MERSHON, D. H. The perception of size in a distorted room. Perception \& Psychophysics, 1968, 4, 26-28.

Gogel, W. C., \& Mertens, H. W. Perceived depth between familiar objects. Journal of Experimental Psychology, 1968, 77. 206-211.

GOGEL, W. C., \& NEWTON, R. E. Perception of off-sized objects. Perception \& Psychophysics, 1969, 5, 7-9.

Newman, C. V. Familiar and relative size cues and surface texture as determinants of relative distance judgments. Journal of Experimental Psychology, 1972, 96, 37-42.

ONo, H. Apparent distance as a function of familiar size. Journal of Experimental Psychology, 1969, 79, 109-115.

Schiffman, H. R. Size estimation of familiar objects under informative and reduced conditions of viewing. American Journal of Psychology, 1967, 80, 229-235.

Slack, C. W. Familiar size as a cue to size in the presence of conflicting cues. Joumal of Experimental Psychology, 1956. 52, 194-198.

\section{NOTE}

1. This is not to claim that such "give away" cues might not occur (and be crucial) in certain other circumstances. For example, it would be foolish to argue that the presentation of a normally active object, e.g., a human being, as a static transparency would have the necessary character to be seen as the intended familiar object. Realistic presentations of this kind probably can only be achieved at present by the use of an actual person (cf. Carlson \& Tassone, 1971). Fortunately, however, one may avoid this particular problem by using representations of normally inanimate objects. preferably that objects which are commonly seen in the frontoparallel position in which they are to be presented.

(Received for publication August 22, 1974; revision received September 20, 1974.) 